\title{
Meeting Report: University of Wisconsin/ AAPS/FDA Workshop-Applied Biopharmaceutics and Quality by Design for Dissolution/Release Specification Setting: Product Quality for Patient Benefit

\author{
Vivian A. Gray \\ Dissolution Technologies \\ Hockessin, DE
}

A meeting on Dissolution and Quality by Design $(\mathrm{QbD})$, co-sponsored by Extension Services in Pharmacy, School of Pharmacy, University of Wisconsin and FDA in cooperation with AAPS, was held June 10-12, 2009, in Rockville, MD.

In the first talk, "Impact of $\mathrm{QbD}$ and Future Expectations: Role of Biopharmaceutics and Quality by Design for in vitro Dissolution/Release Specifications Setting," Helen Winkle of FDA stated that the future of $C M C$ review is $Q b D$ and related tools that will allow companies to build better quality into their products. Significant scientific and technological advances are facilitating improved bridging of product development and manufacturing to clinical relevance. She asked, as we gain momentum from the new QbD paradigm, what would be new and unique opportunities from early integration of biopharmaceutics? An outcome of the conference could be a better understanding of the relationship between biopharmaceuticals and $\mathrm{QbD}$ and how the integration can improve the regulatory process.

The second speaker was Arzu Selen, also of FDA, who spoke on "Introduction to the Conference Workshop Objectives, Program Contents and Strategy: Clinically Meaningful in vitro Drug Release/Dissolution Specification Setting as Guided by Optimized use of Biopharmaceutics and the QbD Tools." She put forth four points to consider during the workshop: how to determine what is relevant or critical for patient benefit; what steps to take for "success" of a drug product; what are the "right" tools for the "right" studies; and what is the impact of "timing." She reiterated that there is a clear shift of expectations in drug dissolution-release testing, from providing basic criteria for product drug release, to providing batch-to-batch consistency, to being a potential surrogate for in vivo $B E$ studies, and finally, to linking the product and its in vivo performance. She laid out the path to integration QbD and biopharmaceutics and summarized with the iterative process of building on knowledge gained to optimize the product. Dissolution testing should be predictive, and methods should be discriminatory with specifications linked to product critical quality attributes and intended in vivo performance.

\author{
Plenary Session 1: Defining the First Principles in \\ Selecting and Developing the "Candidate" \\ The first speaker was Anette Mullertz, Director of
} The Bioneer: FARMA Center of the University of Copenhagen. Her topic was Vision for Now and the Future: Preclinical Development Utilizing Biopharmaceutics and QbD Principles can Guide, Support, and Optimize in vivo Drug Performance. She first discussed the BCS and BDDCS as a way of classifying drug substances along with identifying the limiting factors for absorption. The composition of human intestinal fluids was explained with references from the literature. She suggested that the fed intestinal fluid should contain mono-olein glycerides and sodium oleate fatty acids. A case study using Danazol and other poorly soluble drugs was presented, showing that IVIVC could only be obtained with the use of monoglycerides and fatty acids in the media. An in vitro lipolysis model was shown. She concluded by calling for more predictive models for poorly soluble drugs.

Maria Cruanes from Merck was the second speaker. She discussed in vitro tools to guide early development of low-solubility compounds: critical excipients and critical API attributes. The focus of her talk was the early capture and documentation of knowledge of the critical quality attributes. Relative to the Target Product Profile, there should be enhanced in vivo exposure as an important early objective in development. The in vitro screening of excipients can guide excipient selection and help identify the critical API and formulation attributes and increase the probability of success in vivo. In particular, notice the excipients ability to solubilize or disperse the API and use miniaturization whenever possible. She suggested the use of a "modified" sink condition to get a discriminating test. The use of unconventional tools to gain understanding of the release mechanism and in silico models was encouraged.

Tahseen Mirza from Novartis finished the session with a talk titled, "Value of Risking-Taking and Lessons Learned from Utilization of in vitro and Animal Models for Risk 


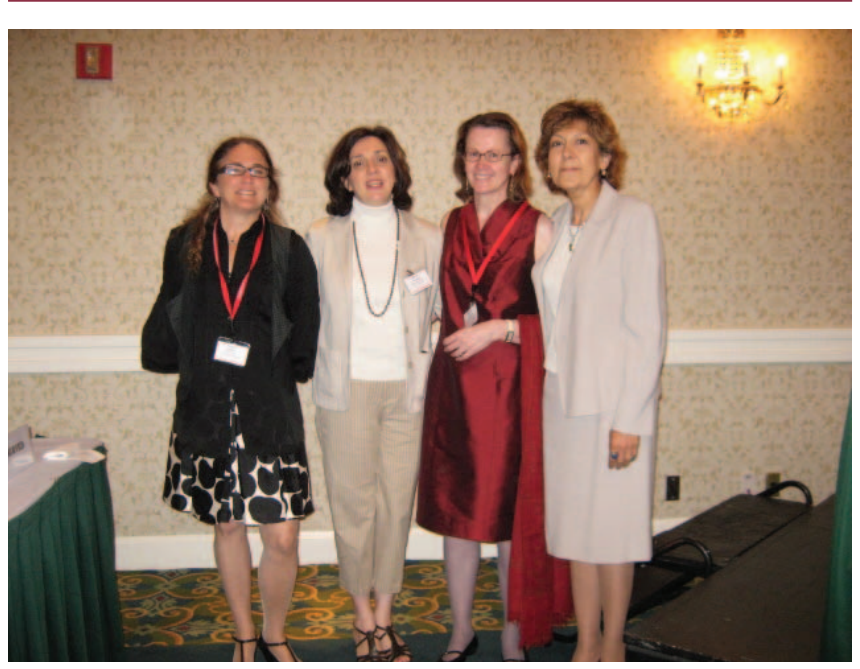

Planning Committee: Anette Mullertz, Maria Cruanes, Jennifer Dressman, Arzu Selen, Jim DeMuth (not pictured).

Assessment During Insoluble Compound Formulation Development." He pointed out that the real dilemma is how you determine if the batches that fall within and outside the design space have any clinical relevance. He suggests that this is done by introducing in vivo data through animal exposure at the earliest phases and utilizing rank order as a preliminary tool. The use of micro dissolution apparatus is very worthwhile in early phases where the API supply is limited.

Plenary Session 2: Utilizing Biopharmaceutics Tools to Design and Guide Drug Delivery for Efficacy and Safety; How Can Biopharmaceutics and QbD Support the Clinical Outcome and Aid in Setting Clinically Meaningful in vitro Dissolution/Release Specifications?

The first talk in the second session was given by Paul Dickinson of AstraZeneca. His topic was "How Can in vitro Data Aid Specification Setting and Guide Design Space Development in an Approach Consistent with QbD: The Role of Drug Substance Physicochemical Properties; BCS Class and Clinical Data." He charged that methods should be developed that give product-specific knowledge relating predominately to safety and efficacy. This could be done in two ways: (1) a priori "biorelevant" dissolution methodologies and (2) development of dissolution conditions and a specification that guarantee drug exposure (safety and efficacy) is the same as that in the pivotal trails and will still be able to be operated as a traditional quality control method. He presented several case studies.

The next speaker was Gordon Muirhead from GlaxoSmithKline, and he spoke about understanding and integrating design space and biopharmaceutics that will be adequate to assure product quality from the standpoint of bioperformance. He discussed establishing a QbD specification. In order to do this, the Critical Quality Attributes (CQAs) should be linked to "clinical relevance" by virtue of their impact on efficacy, safety, or reproducible performance. Clinically relevant CQAs should be linked to Critical Process Parameters (CPPs) either directly or indirectly. One or more CPP, which controls the "clinically relevant" CQA, could be enabled by real-time monitoring or process analytical technology (PAT). The acceptance criteria should be defined by dimensional relationships between CPPs and CQAs in a manner that enables the operational criteria to be contained with a design space.

The third speaker of the session was Jack Cook from Pfizer, whose topic was to consider pre- and post-approval manufacturing changes for a drug, asking what would be an efficient QbD path for exploring the potential impact of formulation (or dosage form) changes on in vivo performance. He started by observing that recent techniques and advances have allowed pharmaceutical scientists to measure the impact of changes in process and materials on in vitro formulation performance. However, our ability to interpret the relevance of any effect is often challenged by a lack of understanding on how in vitro changes are reflected in vivo. How does one establish this link between dissolution and in vivo performance? One point was for the scientist to become less of a frequentist-using fewer assumptions with individual study focus to a more Bayesian view: learn from previous studies and use DOE frequently. Now IVIVC is still avoided, but the reality is without one, there will always be another BE study, slowing down the development. FDA is increasingly requesting companies to do an IVIVC. He summarized by stating that the BCS system can be used to determine the goal of assessment of the relationship between the dissolution test and product performance. With high-solubility drugs, there is a region of bioequivalence, and with low-solubility drugs, an IVIVC is possible. For products reaching the market, the IVIVC will pay for itself. He also mentioned that current software greatly facilitates IVIVC development.

Arzu Selen finished the session with the topic of bridging product critical quality attributes (CQAs) to in vivo performance and clinical outcomes. In her presentation, Arzu emphasized the application of biopharmaceutics to build a bridge between the pharmaceutical product and the patient. Her proposed tools are the Quality Target Product Profile (QTPP), CQA, and the Clinical Outcome. Her approach is more "outside the box" by exploring new opportunities for patient benefit.

Considering the Critical Quality Attributes in terms of pharmaceutical development as defined by ICH Q8R1, the design space in terms of pharmaceutical manufacturing, dissolution testing can be used to make the transition 
to the Quality by Design approach. The goal here is to link the design space with the in vivo performance parameters. One possible approach Arzu mentioned is the Design of Experiments, which can be used for the transition of the traditional dissolution testing to $\mathrm{QbD}$.

Arzu used two case studies to illustrate her approach. The first described the case where the properties of the active ingredient were well known. The drug product should be a chronotherapeutic formulation achieving the maximum concentration in the morning. It was shown that the desired delayed release profile could be achieved with a certain $\mathrm{API} /$ polymer ratio. Further formulation development was made using in vitro and in silico tools. The developed formulation was then evaluated in a preclinical study. The second case study used an early stage development example. In this case, predictive mathematical modeling of in vitro drug release profiles was used to achieve the desired in vivo response.

With these two case studies, Arzu highlighted that critical quality attributes based on science- and risk-based approaches can be linked to the clinical outcome.

\section{Plenary Session 3: Defining "Predictive" and Identifying Useful Tools: How Predictive "Predictive" Needs To Be for Ensuring Product Quality, Safety, and Efficacy? Innovative, Advanced or Borrowed Tools: Pros and Cons}

The session began with Jennifer Dressman of J. W. Goethe University speaking on acceptable predictive and biopharmaceutics tools. She stated our current tools of biowaivers and IVIVC to ensure batch-to-batch bioequivalence and posed the question, "Where are the gaps and can biopharmaceutics tools fill the gaps?" With the $\mathrm{QbD}$ paradigm within the design space, all batches are bioequivalent, and PK studies to demonstrate BE are unnecessary. To achieve this goal, we need to link QbD parameters to in vivo performance through biopharmaceutics tools. These tools are necessary, as it would be impractical to define the importance of each component and manufacturing step through PK studies. She summarized the tools by encouraging to keep it simple whenever possible: use biowaivers for highly soluble drugs and for poorly soluble drugs in IR formulations, use media that are likely to reflect the in vivo performance. She suggested that physiologically based pharmacokinetic models (PBPK) can extend the biopharmaceutics repertoire and asked that deviation from the $80-125$ rule be tolerated at times.

The second speaker was Christos Reppas from the National and Kapodistrian University of Athens, who spoke on the topic "Can in vitro Methods/Tools Adequately Predict in vivo Food Effect and/or Drug Absorption Under Extreme Conditions or in Special Populations?" He addressed the unique capabilities of biorelevant media. This type of media can identify the scope of solubility problems after oral administration and also identify suitable formulation strategies. Biorelevant media can be used to test the robustness of performance to food effects and to elevated gastric $\mathrm{pH}$ (achlorhydric model) and can evaluate precipitation in the small intestine (transfer model).

The third speaker was Filippos Kesisoglou from Merck, who spoke on the pros and cons to moving forward with an industry perspective. He reviewed the existing tools and put forth computational tools as a way to link in vitro tools to clinical performance. He presented some case studies illustrating these points. The advantages of computational tools are that they allow for a mechanistic link of API/formulation properties to bioperformance; enable assessment of multiple "what if" scenarios early on to identify key parameter attributes; allow exploration of scenarios that would be impractical to evaluate in preclinical species or just dissolution assays; and lastly, allow identification of lead options and rational design of clinical studies. The disadvantages of computational tools are that there are approximations in the models; there is difficulty in capturing some of the in vivo absorption processes; validation of models is not always straightforward (what constitutes a validated method?); and finally, as with every modeling approach, there is a general skepticism around modeling.

Stefaan Rossenu of Johnson and Johnson spoke on the use of non-linear, mixed-effects models to establish an IVIVC for a long-acting injectable. He discussed the various IVIVC approaches, study designs, and application of the IVIVC model. For the long-acting injectable, an IVIVC model incorporating the non-linear, mixed-effects modeling approach was developed to describe the in vivo performance. The use of a time-scaling approach was successful. Individual data were used instead of average data. The predictability of the IVIVC model confirmed the biorelevance of the dissolution test and fulfilled the FDA criteria for the internal and external validation. The end result was that three biorelevant in vitro release specifications were proposed, and formulations at the upper and lower ends of the specification were predicted to be well within the accepted bioequivalence range.

The session ended with Patrick Marroum of the FDA. His topic was "Pros and Cons: Tools to Move Forward: A Regulatory Perspective." He stated that the regulatory objective was to optimize therapy to the patient by assuring that the marketed batches have the same safety and efficacy profiles as the ones tested in the clinical trials and to minimize the risk to the patient by decreasing variability from batch to batch. With the advantages of the tools we have already, dissolution can be a surrogate for in vivo performance, we are obtaining a better understanding of factors affecting the performance of the formulations, and clinically relevant specifications are possible. The obstacles are that there is difficulty in manufacturing formulations with different characteristics, 
and development programs could be very expensive. There is also a fear that data generated may be used adversely by FDA. An IVIVC for IR formulations can be difficult because the rate-limiting step is not the release of the API from the formulation. The in vitro dissolution can be over-discriminating and sometimes it is difficult to obtain sensitivity and discriminatory dissolution methods for highly insoluble drugs.

There are also special challenges for the specialized dosage forms. The levels of drug can be too low; the dosing can be of a long-term duration; in vivo studies may be difficult to conduct; the in vitro methods may not be able to reflect in vivo behavior; the dissolution equipment may not be designed for some products; and lastly, the effect is local and not systemic, therefore little value is obtained from plasma levels. He gave several case studies for IVIVC of novel dosage forms. His conclusions centered on encouraging sponsors to prospectively design the appropriate in vitro and in vivo studies to provide a better understanding of the factors that will influence the in vivo performance of the drug product. Lastly, early and frequent interactions with the agency are encouraged.

\section{Breakout Sessions on Approaches/Tools for Setting in vitro Dissolution/Release Specifications and Recommendations for the Next Steps}

The individual breakout sessions were as follows:

1) Integrating Biopharmaceutical Assessment into the QbD Paradigm

2) Predictive Statistical Tools

3) Predictive Mechanistic Tools

4) Predictive Analytical Tools

\section{Plenary Session 4: Merging Old and New: Implementation Challenges}

First, Jobst Limberg from the Federal Institute for Drugs and Medical Devices in Germany presented a regulatory perspective from Europe. He presented the European safety, efficacy, and quality authorization procedures and the concept of bioequivalence. Under the concepts for pharmaceutical quality, he discussed how marketing authorization is based on the description of a model for the drug product, the role of specifications, and the potential role of Process Analytical Technology (PAT). He explored in vitro dissolution test method development and the potential discriminatory power of the test. IVIVC was discussed along with different types of specifications for different dissolution behavior. He suggested that an alternative to characterize the dissolution behavior of a prolonged extended-release drug product could be to specify the dissolution rate over time (e.g., the drug releases $7-13 \%$ [average] of the label claim per hour in an interval between 2 nd and 7 th hour of the in vitro test). He also presented an approach to PAT that included using multivariate data analysis where differences may be detected and using principal component analysis to build clusters of acceptable and unacceptable batches. The nondestructive nature of the very short measuring time makes it possible to enlarge the amount of units tested, and in-process testing may be applicable to control critical manufacturing parameters.

A regulatory perspective from Japan was presented by Chikako Yomota from the National Institute of Health Sciences. She presented the Bioequivalence Guidelines for Oral Dosage Forms in Japan, pointing out the attention that is paid to the $\mathrm{pH}$ range to account for the presence of achlorhydria in the Japanese population. The guidelines do instruct on the use of dissolution as a bioequivalence tool and contain a very useful chart of the paddle speed and media $\mathrm{pH}$ to be used with certain types of drugs (acidic, neutral, basic, low solubility, and coated products). She also gave the regulatory perspective in Japan of QbD, which includes DOE, and presented a case study of a special film-coated tablet, Yokozuan Tablets.

Alan Royce from Novartis gave a pharmaceutical industry perspective. He described the QbD development scheme using biopharmaceutics and QbD in the API, formulation, and final process. He emphasized the need for a team approach that is forward-looking and stressed the importance of DOE. The selection of a risk matrix for API characteristics, formulation platform, and formulation optimization was discussed, and an example of the risk assessment procedure was given. He concluded with how QbD can provide a multivariate understanding of CQAs; that biopharmaceutical properties with $\mathrm{QbD}$ tools can be for NME, salt selection, polymorph selection, and particle size.

The United States regulatory perspective was presented by Christine Moore of the FDA. She outlined a possible QbD approach to dissolution release specification setting. First, develop an initial quality target product profile; perform an initial estimation of the relationships between the CQAs and in vivo performance; determine aspects of the formulation and process that are critical to the release profile; determine the sources of variability and optimize the formulation; and finally, use models to understand potential changes in material and manufacturing operations. She defined Real-Time Release Testing (RTRT) as the ability to evaluate and ensure the quality of in-process and final product based on process data. This approach can be facilitated by having fast assays or surrogate assays for dissolution release testing. She offered alternatives to dissolution testing: the disintegration test and surrogate models. The remaining gaps are computational and experimental methodologies, complex dosage forms, and patient variability. The regulatory aspects of the remaining challenges are the need for increased scientific dialogue, the detail and placement of information in the application, and international acceptance and harmonization. She concluded by stating that QbD approaches can lead to a fundamental paradigm shift for pharmaceutical development and manufacturing, 
providing a linkage between patient, product, and process and a more risk-based approach to regulatory oversight.

The meeting was concluded with the listing of nine priorities to achieve the future state where drug dissolution release specifications are based on desired clinical (in vivo) performance. They are as follows:

1. Multi-dimensional collaboration including multidisciplinary tool development
2. Computational tools

3. Context-specific specifications

4. Database that includes successful and unsuccessful stories

5. Common glossary of terminology

6. Improve manufacturing process

7. Increase mechanistic understanding

8. Appreciation of variability

9. Biopharmaceutics risk-assessment road map

\section{Basic Dissolution Testing: AAPS Short Course on CD-ROM}

\section{About the Course:}

Dissolution testing is a critical test for measuring the performance of a drug product. The dissolution test has been a relatively new analytical technique with equipment modifications and improvements spanning the last decade. The importance of the test has also increased considerably in the last 10 years. It is a quality control tool and an aid to formulation development. Dissolution testing measures change on stability, and is used to establish an in-vitro and in-vivo correlation for some products.

\section{Course Contents:}

- General Overview

\section{- Equipment}

$\underset{\text { Distance Leaming }}{\operatorname{aaph}}$
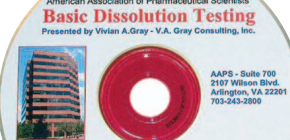

- Sources of Error During Calibration and Sample Testing

- Utility of Dissolution Testing

- Selecting Test Conditions and Method Development

- Method Validation

- Role of USP in Dissolution Testing

- FDA Role in Dissolution and Guidances, Other Regulatory Guidances, and Additional Resources

\section{About the Presenter:}

Vivian A. Gray was the Head of the Dissolution Group in the Analytical Research and Development Section of DuPont Pharmaceuticals Company in Wilmington, Delaware when this presentation was prepared in 2000 . Prior to joining DuPont Pharmaceuticals Company, she was with the United
States Pharmacopeia in Rockville, Maryland. Vivian has spent the last 30 involved in all aspects of dissolution testing, including regulatory (issolution method development and validation, and evaluating new dissolution technology. In 2002, she formed her own consulting company in dissolution testing and related areas. The company name is V. A. Gray, Consulting, Inc., www.vagrayconsulting.net, located in Hockessin, DE. She is now Managing Director of Dissolution Technologies, a www.dissolutiontech.com

\section{Who Should Participate:}

This CD-ROM Short Course is suitable for anyone who must need to understand dissolution and how dissolution tests are performed, including those involved in formulation, QC, R\&D, metrology, and regulation.

\section{Technical Platform and Requirements:}

This two-hour course is contained within a standard CD-ROM. Requirements include a 486 or faster PC running Windows 9x, a CD-ROM player, sound card, and speakers. Included on the disk is a copy of PowerPoint 97 Viewer, which is required to view the course content, and which must be installed in the default directory in order to properly utilize the menu-driven program.

Price:

$\$ 180.00$ domestic, $\$ 200.00$ outside USA. Credit card payment available.

For complete ordering information go online to

www.dissolutiontech.com or contact:

Dissolution Technologies

9 Yorkridge Trail, Hockessin, DE 19707

Phone-302-235-0621, Fax-443-946-1264

Email:vgray@ dissolutiontech.com 